\title{
OPTIMAL HEAT KERNEL BOUNDS UNDER LOGARITHMIC SOBOLEV INEQUALITIES
}

\author{
D. BAKRY, D. CONCORDET, M. LEDOUX
}

\begin{abstract}
We establish optimal uniform upper estimates on heat kernels whose generators satisfy a logarithmic Sobolev inequality (or entropy-energy inequality) with the optimal constant of $\mathbb{R}^{n}$. Off-diagonals estimates may also be obtained with however a smaller distance involving harmonic functions. In the last part, we apply these methods to study some heat kernel decays for diffusion operators on $\mathbb{R}^{n}$ of the type $\Delta-\nabla \cdot \nabla U$ for some smooth potential $U$ with a given growth at infinity.
\end{abstract}

\section{INTRODUCTION AND MAIN RESULT}

Let, for example, $M$ be a Riemannian manifold of infinite volume, and let $d v$ denote its Riemannian volume element. In the study of heat kernel bounds, various functional inequalities have been used in the past years: Sobolev inequalities [Varopoulos (1985)] (with $n>2$ )

$$
\|f\|_{2 n / n-2}^{2} \leq C \int|\nabla f|^{2} d v, \quad f \in C_{c}^{\infty}(M)
$$

logarithmic Sobolev inequalities [Davies (1989)] or entropy-energy inequalities [Bakry (1994)]

$$
\int f^{2} \log f^{2} d v \leq \frac{n}{2} \log \left(C \int|\nabla f|^{2} d v\right), \quad f \in C_{c}^{\infty}(M), \int f^{2} d v=1,
$$

and Nash inequalities [Carlen et al. (1987)]

$$
\|f\|_{2}^{2+4 / n} \leq C\|f\|_{1}^{4 / n} \int|\nabla f|^{2} d v, \quad f \in C_{c}^{\infty}(M) .
$$

As shown in these papers, these three functional inequalities are all equivalent to a common upper bound on the heat kernel $p_{t}(x, y)$ on $M$ given by

$$
\sup _{x, y \in M} p_{t}(x, y) \leq \frac{C^{\prime}}{t^{n / 2}}, \quad t>0 .
$$

In particular, the inequalities (S), (LS) and (N) are equivalent, for possibly different $C>0$ but for the same (analytic) dimension $n(>2$ in case of $(\mathrm{S})$ ).

URL address of the journal: http://www.emath.fr/ps/

Received by the Journal May 17, 1996. Revised May 15, 1997. Accepted for publication September 18, 1997.

(c) Société de Mathématiques Appliquées et Industrielles. Typeset by $\mathrm{T}_{\mathrm{EX}}$. 
This may also be shown directly [Bakry et al. (1995)]. Actually, the previous inequalities belong to a whole family of equivalent inequalities of the type

$$
\|f\|_{r} \leq C\|f\|_{s}^{\theta}\left(\int|\nabla f|^{2} d v\right)^{(1-\theta) / 2}, \quad f \in C_{c}^{\infty}(M)
$$

with

$$
\frac{1}{r}=\frac{\theta}{s}+\frac{1-\theta}{q}, \quad \theta \in[0,1[
$$

and $q=2 n / n-2$. The logarithmic Sobolev inequality (LS) corresponds to the limiting case $r=2$ and $\theta=1$ (cf. [Bakry et al. (1995)]).

When $M=\mathbb{R}^{n}$ with Lebesgue measure $d x$, the best constants in the three inequalities (S), (LS) and (N) are known. Namely

$$
C=\frac{\left(\frac{1}{2}(n+1) !\left|B^{n}\right|\right)^{2 / n}}{\pi^{2} n(n-2)},
$$

where $\left|B^{n}\right|$ denotes the volume of the unit ball $B^{n}$ in $\mathbb{R}^{n}$, in case of (S) [Aubin (1982)],

$$
C=\frac{2((n+2) / 2)^{(n+2) / n}}{n \lambda_{1}^{N}\left|B^{n}\right|^{2 / n}},
$$

where $\lambda_{1}^{N}$ denotes the first non-zero Neumann eigenvalue of the Laplacian on radial functions on $B^{n}$, in case of (N) [Carlen et al. (1993)]. For (LS), the task is easier [Carlen (1991)]. One may simply start with the logarithmic Sobolev inequality [Gross (1975)] for the canonical Gaussian measure $\gamma_{n}$ on $\mathbb{R}^{n}$ with density $\varphi_{n}(x)=(2 \pi)^{-n / 2} \exp \left(-|x|^{2} / 2\right)$, that indicates that, for every smooth function $g$ on $\mathbb{R}^{n}$ with $\int g^{2} d \gamma_{n}=1$,

$$
\int g^{2} \log g^{2} d \gamma_{n} \leq 2 \int|\nabla g|^{2} d \gamma_{n}
$$

Set $f^{2}=\varphi_{n} g^{2}$ so that $\int f^{2} d x=1$. Then

$$
\int f^{2} \log \left(f^{2}(2 \pi)^{n / 2} \mathrm{e}^{|x|^{2} / 2}\right) d x \leq 2 \int\left|\nabla f+\frac{x}{2} f\right|^{2} d x
$$

An integration by parts easily yields

$$
\int f^{2} \log f^{2} d x \leq 2 \int|\nabla f|^{2} d x-\frac{n}{2} \log (2 \pi)-n .
$$

Changing $f$ into $\lambda^{n / 2} f(\lambda x), \lambda>0$, which still satisfies the normalization $\int f^{2} d x=1$, shows that, for every $\lambda>0$ thus,

$$
\int f^{2} \log f^{2} d x \leq 2 \lambda^{2} \int|\nabla f|^{2} d x-\frac{n}{2} \log (2 \pi)-n-n \log \lambda .
$$

Optimizing in $\lambda$, we get that for every smooth $f$ on $\mathbb{R}^{n}$ with $\int f^{2} d x=1$,

$$
\int f^{2} \log f^{2} d x \leq \frac{n}{2} \log \left(\frac{2}{n \pi \mathrm{e}} \int|\nabla f|^{2} d x\right) .
$$


Since we started from the logarithmic Sobolev inequality for $\gamma_{n}$ with its best constant for which exponential functions are extremal,

$$
C=\frac{2}{n \pi \mathrm{e}}
$$

is the best constant in the inequality (LS) on $\mathbb{R}^{n}$ (with respect to Lebesgue measure). To further convince ourselves that this constant is optimal, one may note that among all functions $\Phi: \mathbb{R}^{+} \rightarrow \mathbb{R}^{+}$such that, for every smooth function $f$ on $\mathbb{R}^{n}$ with $\int f^{2} d x=1$,

$$
\int f^{2} \log f^{2} d x \leq \Phi\left(\int|\nabla f|^{2} d x\right)
$$

the function

$$
\Phi(u)=\frac{n}{2} \log \left(\frac{2 u}{n \pi \mathrm{e}}\right)
$$

is actually best possible. Indeed, apply (1.3) to $f^{2}(x)=\lambda^{n} \varphi_{n}(\lambda x), \lambda>0$. Since $\int f^{2} d x=1$, we get

$$
\frac{n}{2} \log \left(\frac{\lambda^{2}}{2 \pi}\right)-\frac{n}{2} \leq \Phi\left(\frac{n \lambda^{2}}{4}\right)
$$

The claim follows by setting $u=n \lambda^{2} / 4$.

Since (S), (LS) and (N) all imply the heat kernel upper bound (1.1), one may wonder if one of these inequalities with the optimal Euclidean constant could yield the optimal Euclidean heat kernel bound

$$
\sup _{x, y \in M} p_{t}(x, y) \leq \frac{1}{(4 \pi t)^{n / 2}}, \quad t>0 .
$$

It is possible that this is the case for the three inequalities. The main result of this note is that this is the case with the logarithmic Sobolev inequality (LS). The proof of this result appears as a consequence of the very precise study of uniform upper estimates on the heat kernel under functional inequalities between entropy and energy developed in [Bakry (1994)]. We present below this result in the context of abstract Markov semigroups of [Bakry (1994)]. We obtain by the same method the sharp bounds on the norm $\left\|P_{t}\right\|_{p, q}$ of the heat semigroup on $\mathbb{R}^{n}$ as an operator from $\mathrm{L}^{p}$ into $\mathrm{L}^{q}$. Various comments complement this first section. In particular, we deduce from our main result that a complete Riemannian manifold of dimension $n$ with non-negative Ricci curvature that satisfies (LS) with the best constant of $\mathbb{R}^{n}$ is necessarily isometric to $\mathbb{R}^{n}$. In Section 2, we discuss the off-diagonal upper bounds on the heat kernel. We namely observe that, again with the methods of [Bakry (1994)], we may obtain the optimal estimates provided the distance is replaced by a smaller one that takes into account the structure of harmonic functions. In the last section, we use the tool of functional inequalities between entropy and energy to study diffusion operators on $\mathbb{R}^{n}$ defined by $\mathrm{L} f=\Delta f-\nabla f \cdot \nabla U$ for some potential $U$. According to the growth of $U$ at infinity, we obtain various heat kernel estimates that extend 
and clarify some of the results of [Kavian et al. (1993)]. After this work was completed, we became aware of the recent paper [Carlen et al. (1995)] by E. Carlen and M. Loss where the authors obtain optimal estimates for viscously damped conservation laws also relying on Gross's method and on the sharp logarithmic Sobolev inequality on $\mathbb{R}^{n}$. They do not consider however the general implication we establish in Theorem 1.2 below.

We first turn to (1.4) and describe, to begin with, the framework in which we will formulate most of our results, refering to [Bakry (1994)] for further details. On some measure space $(E, \mathcal{E}, \mu)$, let L be a Markov generator associated to some semigroup $P_{t} f(x)=\int f(y) p_{t}(x, y) d \mu(y)$ continuous in $\mathrm{L}^{2}(\mu)$. We will assume that $L$ and $\mathbb{P}$ are invariant and symmetric with respect to $\mu$. We assume furthermore that we are given a nice algebra $\mathcal{A}$ of (bounded) functions on $E$ dense in the $\mathrm{L}^{2}$-domain of L, stable by $\mathrm{L}$ and $P_{t}$ and by the action of $C^{\infty}$ functions which are zero at zero. (The stability by $P_{t}$ may not be satisfied even in basic examples such as non-degenerate second order differential operators with no constant term on a smooth manifold. This assumption is however not strictly necessary and is mostly used for convenience in order to unify the treatment of a number of various cases that would require each time a separate standard analysis.) We will mainly be concerned here with the case when $\mu$ is infinite. (Although [Bakry (1994)] is mainly concerned with finite measure spaces, all the results from [Bakry (1994)] used here immediately extend to arbitrary measure spaces.) We denote by $\left\|P_{t}\right\|_{p, q}, 1 \leq p<q \leq \infty$, the operator norm of $P_{t}$ from $\mathrm{L}^{p}(\mu)$ into $\mathrm{L}^{q}(\mu)$. Note that

$$
\left\|P_{t}\right\|_{1, \infty}=\sup p_{t}(x, y)
$$

where the supremum is understood in the ess sup sense.

We may introduce, following P.-A. Meyer, the so-called "carré $d u$ champ" operator $\Gamma$ as the symmetric bilinear operator on $\mathcal{A} \times \mathcal{A}$ defined by

$$
2 \Gamma(f, g)=\mathrm{L}(f g)-f \mathrm{~L} g-g \mathrm{~L} f, \quad f, g \in \mathcal{A} .
$$

Note that $\Gamma(f, f) \geq 0$. We will say that $\mathrm{L}$ is a diffusion if for every $C^{\infty}$ function $\Psi$ on $\mathbb{R}^{k}$, and every finite family $F=\left(f_{1}, \ldots, f_{k}\right)$ in $\mathcal{A}$,

$$
\mathrm{L} \Psi(F)=\nabla \Psi(F) \cdot \mathrm{L} F+\nabla \nabla \Psi(F) \cdot \Gamma(F, F) .
$$

This hypothesis essentially expresses that $L$ is a second order differential operator with no constant term and that we have a chain rule formula for $\Gamma, \Gamma(\Psi(f), g)=\Psi^{\prime}(f) \Gamma(f, g), f, g \in \mathcal{A}$. By the diffusion and invariance properties,

$$
\int \Psi(f)(-\mathrm{L} f) d \mu=\int \Psi^{\prime}(f) \Gamma(f, f) d \mu, \quad f \in \mathcal{A} .
$$

One basic operator is the Laplace-Beltrami operator $\Delta$ on a complete connected Riemannian manifold $M$. In the preceding setting, the "natural" measure $\mu$ is only determined from the Riemannian volume element $d v$ up to a constant $d \mu=c d v$. For $\mathcal{A}$ the class, say, of $C_{c}^{\infty}$ functions on $M$ (that is however not stable by the heat semigroup in the non-compact case), 
$\Gamma(f, f)$ is simply the Riemannian length (squared) $|\nabla f|^{2}$ of the gradient $\nabla f$ of $f \in \mathcal{A}$. The previous abstract framework includes a number of further examples of interest (cf. [Bakry (1994)]). For example, one may consider $\mathrm{L}=\Delta+X$ where $X$ is a smooth vector field on $M$, or more general second order differential operators with no constant term. We may also consider infinite dimensional examples such as the Ornstein-Uhlenbeck generator on $\mathbb{R}^{n} \mathrm{~L} f(x)=\Delta f(x)-x \cdot \nabla f(x)$.

In this setting, and following [Bakry (1994)], one may consider general inequalities between the entropy $\int f^{2} \log f^{2} d \mu$ and the energy $\int \Gamma(f, f) d \mu$ of a function $f$ in $\mathcal{A}$ with $\int f^{2} d \mu=1$. Assume that, for some $\Phi: \mathbb{R}^{+} \rightarrow \mathbb{R}^{+}$, for every $f \in \mathcal{A}$ with $\int f^{2} d \mu=1$,

$$
\int f^{2} \log f^{2} d \mu \leq \Phi\left(\int \Gamma(f, f) d \mu\right)
$$

In most examples $\Phi$ is concave, strictly increasing, and of class $C^{1}$, which we assume throughout the argument below. Therefore, for every $v>0$, $\Phi(u) \leq \Phi(v)+\Phi^{\prime}(u)(u-v)$, so that (1.5) reads, for every $v>0$ and every $f$ (and by homogeneity),

$$
\int f^{2} \log f^{2} d \mu \leq \Phi^{\prime}(v) \int \Gamma(f, f) d \mu+\Psi(v) \int f^{2} d \mu
$$

where $\Psi(v)=\Phi(v)-v \Phi^{\prime}(v)$. We thus deal equivalently with a family of logarithmic Sobolev inequalities. Now, by the diffusion property, changing $f>0$ into $f^{p / 2}$ shows that, for every $f>0, v>0, p>1$,

$$
\begin{aligned}
& \int f^{p} \log f^{p} d \mu-\int f^{p} d \mu \log \int f^{p} d \mu \\
& \leq-\Phi^{\prime}(v) \frac{p^{2}}{4(p-1)} \int f^{p-1} \mathrm{~L} f d \mu+\Psi(v) \int f^{p} d \mu .
\end{aligned}
$$

Choose now in this inequality a function $v \rightarrow v(p)>0, p>1$. According to [Bakry (1994)], we make then use of the fundamental argument of L. Gross [Gross (1975)]. Namely, if

$$
V(t)=\mathrm{e}^{-m(t)}\left\|P_{t} f\right\|_{p(t)}, \quad t \geq 0,
$$

the preceding inequality will show that $V^{\prime} \leq 0$ and $V$ is non-increasing as soon as $p$ and $m$ are chosen so that

$$
\frac{p^{2}(t)}{p^{\prime}(t)}=\Phi^{\prime}(v(p(t))) \frac{p^{2}(t)}{4(p(t)-1)} \quad \text { and } \quad m^{\prime}(t)=\Psi(v(p(t))) \frac{p^{\prime}(t)}{p(t)^{2}} .
$$

If this is the case, for every $1 \leq p<q \leq \infty$,

$$
\left\|P_{t}\right\|_{p, q} \leq \mathrm{e}^{m}
$$

where

$$
t=t_{p, q}=\int_{p}^{q} \Phi^{\prime}(v(s)) \frac{d s}{4(s-1)} \quad \text { and } \quad m=m_{p, q}=\int_{p}^{q} \Psi(v(s)) \frac{d s}{s^{2}}
$$


provided we can find a function $v$ for which these two integrals are finite. The optimal choice, that will be used throughout this work (cf. [Bakry (1994)]), is given by $v(s)=\lambda s^{2} /(s-1)$, where $\lambda>0$ is a parameter.

It might be worthwhile noting that, conversely, the previous bounds on $\left\|P_{t}\right\|_{p, q}$ imply that the corresponding entropy-energy inequality (1.5) holds. This is a consequence of the following proposition.

Proposition 1.1. Under the previous notation, assume that, for some $1 \leq$ $p<\infty$ and every $q$ in some neighborhood of $p,\left\|P_{t}\right\|_{p, q} \leq \mathrm{e}^{m}$ where $t$ and $m$ are defined with (1.6b) through some function $\Phi$. Then, for every nonnegative $f$ in $\mathcal{A}$,

$$
\begin{aligned}
& \int f^{p} \log f^{p} d \mu-\int f^{p} d \mu \log \int f^{p} d \mu \\
& \leq-\Phi^{\prime}(v) \frac{p^{2}}{4(p-1)} \int f^{p-1} \mathrm{~L} f d \mu+\Psi(v) \int f^{p} d \mu .
\end{aligned}
$$

The proof reduces to check that if, for $f>0$ in $\mathcal{A}$,

$$
U(\varepsilon)=\mathrm{e}^{-m(\varepsilon)}\left\|P_{t(\varepsilon)} f\right\|_{p+\varepsilon}
$$

where $t(\varepsilon)=t_{p, p+\varepsilon}, m(\varepsilon)=m_{p, p+\varepsilon}$, then $U^{\prime}(0) \leq 0$ amounts to the inequality of the proposition.

The main observation of this work is that the general method leading to the bound (1.6) yields the optimal heat kernel bound (1.4) if we start from a (LS) inequality for the generator L (or rather the carré du champ $\Gamma$ ) with the best constant of $\mathbb{R}^{n}$.

Theorem 1.2. Assume that for every $f$ in $\mathcal{A}$ with $\int f^{2} d \mu=1$,

$$
\int f^{2} \log f^{2} d \mu \leq \frac{n}{2} \log \left(\frac{2}{n \pi \mathrm{e}} \int \Gamma(f, f) d \mu\right) .
$$

Then,

$$
\sup p_{t}(x, y) \leq \frac{1}{(4 \pi t)^{n / 2}}, \quad t>0
$$

(where the supremum is understood in the ess sup sense).

If

$$
\int f^{2} \log f^{2} d \mu \leq \frac{n}{2} \log \left(C \int \Gamma(f, f) d \mu\right), \quad \int f^{2} d \mu=1,
$$

for some $C>0$, by a simple change of variables,

$$
\sup p_{t}(x, y) \leq\left(\frac{n e C}{8 t}\right)^{n / 2}, \quad t>0 .
$$

It is worthwhile mentioning that the logarithmic Sobolev inequality (1.7) of Theorem 1.2 behaves correctly under tensor product. Namely, if two carrés $d u$ champ $\Gamma_{1}$ and $\Gamma_{2}$ satisfies such an inequality with dimensions $n_{1}$ and $n_{2}$ respectively, then the product operator $\Gamma_{1} \otimes \Gamma_{2}$ will satisfy this inequality with dimension $n_{1}+n_{2}$. This immediately follows from the classic product 
property of entropy together with the linearized version of (1.7) (the inequality leading to $(1.2)$ ). This stability property is reflected similarly on the heat kernel bound as can be seen from the example of the Euclidean spaces.

Proof. We simply use (1.6) with

$$
\Phi(u)=\frac{n}{2} \log \left(\frac{2 u}{n \pi \mathrm{e}}\right) .
$$

Hence $\Phi^{\prime}(u)=n / 2 u$ and

$$
\Psi(u)=\frac{n}{2} \log \left(\frac{2 u}{n \pi \mathrm{e}^{2}}\right)
$$

Then $\left\|P_{t}\right\|_{1, \infty} \leq \mathrm{e}^{m}$ with

$$
t=t(\lambda)=\frac{n}{8 \lambda} \int_{1}^{\infty} \frac{d s}{s^{2}}=\frac{n}{8 \lambda}
$$

and

$$
m=m(\lambda)=\frac{n}{2} \int_{1}^{\infty} \log \left(\frac{2 \lambda}{n \pi \mathrm{e}^{2}} \cdot \frac{s^{2}}{s-1}\right) \frac{d s}{s^{2}}
$$

where $\lambda>0$. From the first equality, $\lambda=n / 8 t$. The second yields

$$
\begin{aligned}
m & =\frac{n}{2} \log \left(\frac{2 \lambda}{n \pi \mathrm{e}^{2}}\right)+\frac{n}{2} \int_{1}^{\infty} \log \left(\frac{s^{2}}{s-1}\right) \frac{d s}{s^{2}} \\
& =\frac{n}{2} \log \left(\frac{2 \lambda}{n \pi \mathrm{e}^{2}}\right)-\frac{n}{2} \int_{0}^{1} \log (r(1-r)) d r \\
& =\frac{n}{2} \log \left(\frac{2 \lambda}{n \pi \mathrm{e}^{2}}\right)+n
\end{aligned}
$$

with the change of variable $r=1 / s$. Since $\lambda=n / 8 t$,

$$
m=m(\lambda)=\frac{n}{2} \log \left(\frac{1}{4 \pi t}\right)
$$

which yields

$$
\left\|P_{t}\right\|_{1, \infty} \leq \mathrm{e}^{m}=\frac{1}{(4 \pi t)^{n / 2}}
$$

and the result. The proof is complete.

It is clear that the same proof yields upper bounds for $\left\|P_{t}\right\|_{p, q}$ for every $t>0$ and $1 \leq p<q \leq \infty$. Namely $\left\|P_{t}\right\|_{p, q} \leq \mathrm{e}^{m}$ where

$$
t=t_{p, q}(\lambda)=\frac{n}{8 \lambda} \int_{p}^{q} \frac{d s}{s^{2}}=\frac{n}{8 \lambda}\left(\frac{1}{p}-\frac{1}{q}\right)
$$

and

$$
m=m_{p, q}(\lambda)=\frac{n}{2} \int_{p}^{q} \log \left(\frac{2 \lambda}{n \pi \mathrm{e}^{2}} \cdot \frac{s^{2}}{s-1}\right) \frac{d s}{s^{2}} .
$$


After some calculations very similar to the previous ones, we find that

$$
\left\|P_{t} f\right\|_{p, q} \leq\left[\frac{p^{\frac{1}{p}}\left(1-\frac{1}{p}\right)^{1-\frac{1}{p}}}{q^{\frac{1}{q}}\left(1-\frac{1}{q}\right)^{1-\frac{1}{q}}}\right]^{\frac{n}{2}}\left[\frac{1}{4 \pi t}\left(\frac{1}{p}-\frac{1}{q}\right)\right]^{\frac{n}{2}\left(\frac{1}{p}-\frac{1}{q}\right)}
$$

It is less clear however why these bounds should be optimal on $\mathbb{R}^{n}$. The next proposition answers this question positively. These bounds (1.8) and their optimality may also be shown to follow from the work of E. Lieb [Lieb (1990)] on Gaussian maximizers of Gaussian kernels.

Proposition 1.3. With the preceding notation, let $\Phi$ be such that (1.5) holds and $\left\|P_{t}\right\|_{1, \infty}=\mathrm{e}^{m}$ where

$$
t=\int_{1}^{\infty} \Phi^{\prime}\left(\frac{\lambda s^{2}}{s-1}\right) \frac{d s}{4(s-1)}
$$

and

$$
m=\int_{1}^{\infty} \Psi\left(\frac{\lambda s^{2}}{s-1}\right) \frac{d s}{s^{2}}
$$

for some $\lambda>0$. Then, for every $1 \leq p<q \leq \infty$,

$$
\left\|P_{t_{p, q}}\right\|_{p, q}=\mathrm{e}^{m_{p, q}}
$$

where

$$
t_{p, q}=\int_{p}^{q} \Phi^{\prime}\left(\frac{\lambda s^{2}}{s-1}\right) \frac{d s}{4(s-1)}
$$

and

$$
m_{p, q}=\int_{p}^{q} \Psi\left(\frac{\lambda s^{2}}{s-1}\right) \frac{d s}{s^{2}} .
$$

The proof of the proposition is easy. By the hypothesis,

$$
\left\|P_{t_{1, p}+t_{p, q}+t_{q, \infty}}\right\|_{1, \infty}=\left\|P_{t}\right\|_{1, \infty}=\mathrm{e}^{m}=\mathrm{e}^{m_{1, p}+m_{p, q}+m_{q, \infty}} .
$$

But now also, by the semigroup property,

$$
\begin{aligned}
& \left\|P_{t_{1, p}+t_{p, q}+t_{q, \infty}}\right\|_{1, \infty} \leq\left\|P_{t_{1, q}}\right\|_{1, q}\left\|P_{t_{p, q}}\right\|_{p, q}\left\|P_{t_{q}, \infty}\right\|_{q, \infty} \\
& \leq \mathrm{e}^{m_{1, p}+m_{p, q}+m_{q, \infty}}
\end{aligned}
$$

so that it is impossible that $\left\|P_{t_{p, q}}\right\|_{p, q}<\mathrm{e}^{m_{p, q}}$ for some $p<q$. This result clearly applies in the Euclidean case to prove that (1.8) are actually equalities in this case. It moreover implies the somewhat surprising following observation. For every $1 \leq p<q<r \leq \infty$, and every $t \geq 0$, there is an unique $s$ such that

$$
\left\|P_{t}\right\|_{p, r}=\left\|P_{s}\right\|_{p, q}\left\|P_{t-s}\right\|_{q, r} .
$$

Indeed, since $\Phi^{\prime}(u)=n / 2 u$ in this case, one may define $\lambda>0$ by

$$
t=t_{p, r}(\lambda)=\int_{p}^{r} \Phi^{\prime}\left(\frac{\lambda s^{2}}{s-1}\right) \frac{d s}{4(s-1)}=\frac{n}{8 \lambda} \int_{p}^{r} \frac{d s}{s^{2}} .
$$


Set then $s=t_{q, r}(\lambda)$ and the claim follows from the preceding argument.

In order to efficiently use Theorem 1.2 , it would be worthwhile to know how to establish (LS) with sharp constant in a Riemannian or abstract setting using curvature-dimension hypotheses. Before inspecting this question more closely, let us observe the following.

Proposition 1.4. Let $M$ be a Riemannian manifold of dimension $n$ and non-negative Ricci curvature. If, for every non-negative $f$ in $C_{c}^{\infty}(M)$, $\lim _{t \rightarrow \infty}(4 \pi t)^{n / 2} P_{t} f=c \int f d v$, then the heat kernel $p_{t}(x, y)$ on $M$ satisfies

$$
\sup _{x, y \in M} p_{t}(x, y) \leq \frac{c}{(4 \pi t)^{n / 2}}, \quad t>0 .
$$

Proof. It is an immediate consequence of the Li-Yau inequality [Li et al. (1986)] for Riemannian manifolds with non-negative Ricci curvature, that ensures in particular that, if $f>0$,

$$
-\frac{\Delta P_{t} f}{P_{t} f} \leq \frac{n}{2 t}
$$

for every $t>0$ (where $P_{t}$ denotes the heat semigroup on $M$ ). In another words, at every point, the function $t^{n / 2} P_{t} f$ is increasing in $t$. But then, $(4 \pi t)^{n / 2} P_{t} f$ increases to $c \int f d \mu$ so that

$$
\left\|P_{t}\right\|_{1, \infty} \leq \frac{c}{(4 \pi t)^{n / 2}} .
$$

The proof is complete. Note that by the results of P. Li [Li (1986)], $M$ is isometric to $\mathbb{R}^{n}$ if $c=1$ (cf. the proof of Corollary 1.6). Observe furthermore that the proposition similarly applies to generators $\Delta-\nabla \log \rho$ for which the results of [Li et al. (1986)] are also available (in which case the hypothesis on non-negative Ricci curvature has to be replaced by the condition $\Gamma_{2}(f, f) \geq$ $\frac{1}{n}(\mathrm{~L} f)^{2}$ as explained below).

What we actually conjecture, is that if $\lim _{t \rightarrow \infty}(4 \pi t)^{n / 2} P_{t} f=c \int f d v$ for every smooth $f$ on a manifold $M$ of dimension $n$ and non-negative Ricci curvature, then the logarithmic Sobolev inequality (LS) holds with its sharp constant from $\mathbb{R}^{n}$, i.e.

$$
\int f^{2} \log f^{2} d v \leq \frac{n}{2} \log \left(\frac{2 c^{2 / n}}{n \pi \mathrm{e}} \int|\nabla f|^{2} d v\right), \quad \int f^{2} d v=1
$$

(Proposition 1.4 would then be a consequence of Theorem 1.2.) We have not been able to prove such a result although we strongly conjecture that it must be true. So far, we have only been able to prove the inequality with a constant that misses the optimal one by a factor $1 / \log 2$. We present this result in the context of the abstract geometry of Markov generators. Introduce the "iterated carré du champ operator" by setting, for every $f, g$ in $\mathcal{A}$,

$$
2 \Gamma_{2}(f, g)=\mathrm{L} \Gamma(f, g)-\Gamma(f, \mathrm{~L} g)-\Gamma(g, \mathrm{~L} f) .
$$


We say that $\mathrm{L}$ is of curvature $R \in \mathbb{R}$ and dimension $n \geq 1$ if for every $f$ in $\mathcal{A}$,

$$
\Gamma_{2}(f, f) \geq R \Gamma(f, f)+\frac{1}{n}(\mathrm{~L} f)^{2} .
$$

By Bochner's formula, the Laplace-Beltrami operator on a Riemannian manifold of dimension $n$ and Ricci curvature bounded below by $R$ is of curvature $R$ and dimension $n$ in the preceding functional sense.

Proposition 1.5. Let L be a diffusion generator of curvature 0 and dimension $n \geq 1$. Assume that, for every $f$ in $\mathcal{A}$ with $\int f d \mu=1$,

$$
\limsup _{t \rightarrow \infty} \int P_{t} f \log \left((4 \pi t)^{n / 2} P_{t} f\right) d \mu \leq-\alpha \frac{n}{2}
$$

for some $\alpha>0$. Then, for every $f \in \mathcal{A}$ with $\int f^{2} d \mu=1$,

$$
\int f^{2} \log f^{2} d \mu \leq \frac{n}{2} \log \left(\frac{2}{n \pi \mathrm{e}^{\alpha}} \int \Gamma(f, f) d \mu\right) .
$$

In particular, if (1.9) holds with $\alpha=1$, the inequality holds with the optimal constant of $\mathbb{R}^{n}$.

Proof. It is a simple application of the argument developed in Section 6 of [Bakry (1994)]. Let $f>0$ in $\mathcal{A}$ with $\int f d \mu=1$. By the semigroup properties, for every $t \geq 0$,

$$
\int f \log f d \mu=\int P_{t} f \log P_{t} f d \mu+\int_{0}^{t} F(s) d s
$$

where

$$
F(s)=\int \frac{\Gamma\left(P_{s} f, P_{s} f\right)}{P_{s} f} d \mu .
$$

Now, in the proof of Proposition 6.7 of [Bakry (1994)], it is shown that, under the curvature-dimension assumption on $\mathrm{L}$,

$$
F^{\prime}(s) \leq \frac{2}{n} F(s)^{2}, \quad s \geq 0 .
$$

Hence,

$$
\int_{0}^{t} F(s) d s \leq \frac{n}{2} \log \left(1+\frac{2 t}{n} F(0)\right) .
$$

Together with (1.10) and the assumption on the behavior at infinity of $P_{t}$, the conclusion immediately follows (changing $f$ into $f^{2}$ ). The proof is complete.

What we expect is that actually (1.9) with $\alpha=1$ appears as a consequence of the fact that $\lim _{t \rightarrow \infty}(4 \pi t)^{n / 2} P_{t} f=\int f d \mu$ for every $f$ in $\mathcal{A}$. We only checked so far (1.9) with $\alpha=\log 2$. Let $f$ be non-negative (for simplicity) and such that $\int f d \mu=1$. It is easy to see, by Jensen's inequality, that, for every $t \geq 0$,

$$
\begin{aligned}
\int P_{t} f \log \left((4 \pi t)^{n / 2} P_{t} f\right) d \mu & =\int f P_{t}\left(\log \left((4 \pi t)^{n / 2} P_{t} f\right)\right) d \mu \\
& \leq \int f \log \left((4 \pi t)^{n / 2} P_{2 t} f\right) d \mu
\end{aligned}
$$


In a Riemannian (or concrete) setting, we know (cf. the proof of Proposition 1.4) that $(4 \pi t)^{n / 2} P_{t} f$ is increasing (to 1 ) so that in this case

$$
\limsup _{t \rightarrow \infty} \int P_{t} f \log \left((4 \pi t)^{n / 2} P_{t} f\right) d \mu \leq-\frac{n}{2} \log 2 .
$$

But Proposition 1.5 then only yields

$$
\int f^{2} \log f^{2} d \mu \leq \frac{n}{2} \log \left(\frac{1}{n \pi} \int \Gamma(f, f) d \mu\right), \quad \int f^{2} d \mu=1 .
$$

Note finally that (1.9) with $\alpha=1$ is satisfied in $\mathbb{R}^{n}$. Let $f$ be non-negative on $\mathbb{R}^{n}$ with compact support and such that $\int f d x=1$. Then, for every $x$ in the support of $f$ and every $t$ large enough,

$$
(4 \pi t)^{n / 2} P_{t} f(x)=\int \mathrm{e}^{-|x-y|^{2} / 4 t} f(y) d y \leq 1-\frac{1}{4 t} \int|x-y|^{2} f(y) d y .
$$

Hence,

$$
\log \left((4 \pi t)^{n / 2} P_{t} f(x)\right) \leq-\frac{1}{4 t} \int|x-y|^{2} f(y) d y
$$

Therefore,

$$
\begin{aligned}
P_{t}\left(\log \left((4 \pi t)^{n / 2} P_{t} f\right)\right)(x) & \leq-\frac{1}{4 t} \int \mathrm{e}^{-|x-y-z|^{2} / 4 t}|z|^{2} f(y) d y \frac{d z}{(4 \pi t)^{n / 2}} \\
& =-\frac{1}{4 t} \int\left(|x-y|^{2}+2 t n\right) f(y) d y
\end{aligned}
$$

(1.9) (with $\alpha=1$ ) follows as $t$ tends to infinity.

We conclude this section with a comparison theorem for Riemannian manifolds with non-negative Ricci curvature.

Corollary 1.6. Let $M$ be a complete Riemannian manifold of dimension $n$ with non-negative Ricci curvature satisfying the logarithmic Sobolev inequality (LS) with the best constant of $\mathbb{R}^{n}$, i.e.

$$
\int f^{2} \log f^{2} d v \leq \frac{n}{2} \log \left(\frac{2}{n \pi \mathrm{e}} \int|\nabla f|^{2} d v\right), \quad \int f^{2} d v=1 .
$$

Then $M$ is isometric to $\mathbb{R}^{n}$.

Proof. Denote by $V(x, r)$ the volume of the ball with center $x \in M$ and radius $r>0$ in $M$. By Theorem 1.2 , for every $x, y \in M, t>0$,

$$
p_{t}(x, y) \leq \frac{1}{(4 \pi t)^{n / 2}}
$$

In particular, by the results of [Li et al. (1986)], $M$ has maximal volume growth, that is

$$
\liminf _{r \rightarrow \infty} \frac{V(x, r)}{r^{n}} \geq \theta>0
$$


Li's result $[\mathrm{Li}(1986)]$ then indicates that, for every $x, y, z \in M$,

$$
\lim _{t \rightarrow \infty} V(z, \sqrt{t}) p_{t}(x, y)=\frac{\left|B^{n}\right|}{(4 \pi)^{n / 2}}
$$

where we recall that $\left|B^{n}\right|$ is the volume of the Euclidean unit ball. Together with (1.11), we thus see that

$$
\liminf _{r \rightarrow \infty} \frac{V(x, r)}{\left|B^{n}\right| r^{n}} \geq 1 .
$$

Now, by Gromov's comparison theorem (cf. [Carlen (1991)]), for $s<r$,

$$
\frac{V(x, r)}{\left|B^{n}\right| r^{n}} \leq \frac{V(x, s)}{\left|B^{n}\right| s^{n}}
$$

and, in particular, $V(x, r) \leq\left|B^{n}\right| r^{n}$ as $s \rightarrow 0$. But now, by (1.12), we also get $V(x, s) \geq\left|B^{n}\right| s^{n}$ as $r \rightarrow \infty$. Therefore $V(x, r)=\left|B^{n}\right| r^{n}$ for every $x \in M$ and $r>0$. By the case of equality in the volume comparison theorem, $M$ is isometric to $\mathbb{R}^{n}$. The corollary is established.

\section{OfF-Diagonal ESTimates}

As is well-known [Bakry (1994)], [Davies (1989)], an entropy-energy (or logarithmic Sobolev) inequality of the type (1.5) also leads to off-diagonal upper bounds on the heat kernel in terms of the distance function defined as $d(x, y)=\sup _{\Gamma(f, f)<1}[f(x)-f(y)]$ (the supremum being understood in the ess sup sense).

Let us recall the general procedure of the method due to E. B. Davies (cf. [Davies (1989)]). The idea is to derive from an entropy-energy inequality for the generator $\mathrm{L}$

$$
\int f^{2} \log f^{2} d \mu \leq \Phi\left(\int \Gamma(f, f) d \mu\right), \quad \int f^{2} d \mu=1,
$$

an entropy-energy inequality for $\mathrm{L}^{h}(f)=\mathrm{e}^{-h} \mathrm{~L}\left(\mathrm{e}^{h} f\right)$, where $\Gamma(h, h) \leq \lambda$, which depends only on $\Phi$ and $\lambda>0$. This leads, via the method presented in Section 1, to a uniform upper bound on the kernel $p_{t}^{h}(x, y)=$ $p_{t}(x, y) \mathrm{e}^{h(x)-h(y)}$ of the semigroup $P_{t}^{h}=\mathrm{e}^{-h} P_{t}\left(\mathrm{e}^{h} \cdot\right)$ with generator $\mathrm{L}^{h}$. We thus bound in this way $p_{t}(x, y)$ by

$$
C(t, \delta) \mathrm{e}^{h(x)-h(y)} .
$$

Applying this result to $\lambda h, \Gamma(h, h) \leq 1$, and optimising in $\lambda$ and $h$, leads to some bound

$$
p_{t}(x, y) \leq \mathrm{e}^{V(t, d(x, y))} .
$$

Theorem 5.3 of [Bakry (1994)] yields an explicit form of the function $V$ in terms of the entropy-energy function $\Phi$ of L. Precisely,

$$
p_{t}(x, y) \leq \mathrm{e}^{V(t, d(x, y))} \quad \text { for every } t \leq T(d(x, y))
$$


where the functions $V$ and $T$ are parametrized by $\tau$ and $\theta$ in the following way: if $\tau$ is defined by

$$
d(x, y)=\int_{\tau}^{\infty} \frac{\Phi^{\prime}(s)}{\sqrt{s}} d s
$$

then

$$
T(d(x, y))=\frac{1}{2} \int_{\tau}^{\infty} \frac{\Phi^{\prime}(s)}{\sqrt{s(s-\tau)}} d s,
$$

and if $\theta$ is defined by

$$
t=\frac{1}{2} \int_{\tau}^{\infty} \frac{\Phi^{\prime}(s)}{\sqrt{s(s+\theta-\tau)}} d s
$$

then

$$
V(t, d(x, y))=\sqrt{\frac{\theta}{\tau}} \Phi(\tau)-\frac{\theta-\tau}{2} \int_{\tau}^{\infty} \frac{\Psi(s)}{\sqrt{s^{3}(s+\theta-\tau)}} d s
$$

(where we recall that $\Psi(s)=\Phi(s)-s \Phi^{\prime}(s)$ ).

Let us use these bounds with the optimal entropy-energy function on $\mathbb{R}^{n}$

$$
\Phi(u)=\frac{n}{2} \log \left(\frac{2 u}{n \pi \mathrm{e}}\right), \quad u>0 .
$$

After some computations, we get that

$$
p_{t}(x, y) \leq\left(\frac{\mathrm{e}}{8 n \pi}\right)^{n / 2}\left(\frac{d(x, y)}{t}\right)^{n} \exp \left(-\frac{d(x, y)^{2}}{4 t}\right) .
$$

Unfortunately, this does not give the optimal bound on $\mathbb{R}^{n}$. In order to improve this estimate, we introduce a new distance called the "harmonic" distance defined by

$$
d^{H}(x, y)=\sup _{\Gamma(f, f) \leq 1, \mathrm{~L} f=0}[f(x)-f(y)] .
$$

Note that $d^{H}=d$ on $\mathbb{R}^{n}$ (while $d^{H}=0$ on a compact Riemannian manifold). Proposition 2.1. Assume we have an entropy-energy inequality (1.5). Then

$$
p_{t}(x, y) \leq \exp \left(m-\frac{d^{H}(x, y)^{2}}{4 t}\right)
$$

where $t$ and $m$ are defined in (1.6b) (assumed to be finite).

Proof. The proof follows the same lines as the proof of (1.6a). Fix $h$ with $\mathrm{L} h=0$ and $\Gamma(h, h) \leq 1$ and consider $P_{t}^{\lambda h}=\mathrm{e}^{-\lambda h} P_{t}\left(\mathrm{e}^{\lambda h} \cdot\right)$ whose generator is given by $\mathrm{L}^{\lambda h}(f)=\mathrm{e}^{-\lambda h} \mathrm{~L}\left(\mathrm{e}^{\lambda h} f\right), \lambda>0$. Then, for $f \geq 0$,

$$
\begin{aligned}
-\int f^{p-1} \mathrm{~L}^{\lambda h} f d \mu= & \int \Gamma\left(\mathrm{e}^{-\lambda h} f^{p-1}, \mathrm{e}^{\lambda h} f\right) d \mu \\
= & \int \Gamma\left(f^{p-1}, f\right) d \mu+\lambda(p-2) \int f^{p-1} \Gamma(f, h) d \mu \\
& \quad-\lambda^{2} \int f^{p} \Gamma(h, h) d \mu .
\end{aligned}
$$


The second term on the right-hand-side is equal to

$$
\lambda \frac{p-2}{p} \int \Gamma\left(f^{p}, h\right) d \mu=-\lambda \frac{p-2}{p} \int f^{p} \mathrm{~L} h d \mu=0
$$

so that

$$
-\int f^{p-1} \mathrm{~L}^{\lambda h} f d \mu \geq-\int f^{p-1} \mathrm{~L} f d \mu-\lambda^{2} \int f^{p} d \mu .
$$

Therefore, if, for every $v>0$,

$$
\begin{aligned}
& \int f^{p} \log f^{p} d \mu-\int f^{p} d \mu \log \int f^{p} d \mu \\
& \leq-\Phi^{\prime}(v) \frac{p^{2}}{4(p-1)} \int f^{p-1} \mathrm{~L} f d \mu+\Psi(v) \int f^{p} d \mu,
\end{aligned}
$$

then

$$
\begin{aligned}
& \int f^{p} \log f^{p} d \mu-\int f^{p} d \mu \log \int f^{p} d \mu \\
& \leq-\Phi^{\prime}(v) \frac{p^{2}}{4(p-1)} \int f^{p-1} \mathrm{~L}^{\lambda h} f d \mu \\
& \quad+\left(\Psi(v)+\lambda^{2} \Phi^{\prime}(v) \frac{p^{2}}{4(p-1)}\right) \int f^{p} d \mu .
\end{aligned}
$$

According to the sketch of proof in Section 1, this leads to the upper bound

$$
\left\|P_{t}^{\lambda h}\right\|_{1, \infty} \leq \mathrm{e}^{m+\lambda^{2} t}
$$

where $t$ and $m$ are given by (1.6b). Equivalently,

$$
p_{t}(x, y) \leq \mathrm{e}^{m+\lambda^{2} t-\lambda[h(x)-h(y)]} .
$$

Optimizing in $\lambda>0$, and then in $h$, leads to the claim. Proposition 2.1 is thus established.

As a consequence of this proposition, if

$$
\Phi(u)=\frac{n}{2} \log \left(\frac{2 u}{n \pi \mathrm{e}}\right), \quad u>0,
$$

we get that

$$
p_{t}(x, y) \leq \frac{1}{(4 \pi t)^{n / 2}} \exp \left(-\frac{d^{H}(x, y)^{2}}{4 t}\right)
$$

for every $t>0$ and $x, y$, a formula that is of course optimal on $\mathbb{R}^{n}$. It would be worthwhile to study further examples where $d^{H}=d$, as well as the various bounds which may similarly be deduced through distances involving harmonic functions.

Note also that if we are looking for a situation such that (1.5) holds together with $\left\|P_{t}\right\|_{1, \infty}=\mathrm{e}^{m}$ in (1.6), then

$$
p_{t}(x, y) \leq p_{t}(x, x) \exp \left(-\frac{d^{H}(x, y)^{2}}{4 t}\right)
$$

for every $t>0$ and $x$ such that $p_{t}(x, x)=\sup _{u} p_{t}(u, u)$. 


\section{EXPLICIT BOUNDS FOR SOME LAPLACIAN + POTENTIAL OPERATORS IN EUCliDEAN SPACE}

In this last section, we investigate heat kernel bounds for operators on $\mathbb{R}^{n}$ given by $\mathrm{L} f=\Delta f-\nabla U \cdot \nabla f$ where $U$ is some smooth function whose growth at infinity will determine upper estimates on the heat kernel for small times. The invariant measure is $d \mu=\mathrm{e}^{-U} d x$ (where $d x$ is Lebesgue measure on $\mathbb{R}^{n}$ ) and $\Gamma(f, f)=|\nabla f|^{2}$. The prime example is $U(x)=|x|^{2}$ which is associated to the Ornstein-Uhlenbeck operator. This operator is however only hypercontractive and not ultracontractive (i.e. $\sup p_{t}(x, y)$ is unbounded). We will actually be concerned with functions $U$ that growth faster than $|x|^{2}$ at infinity. Although we work for simplicity with the Laplace operator $\Delta$ on $\mathbb{R}^{n}$, the results would hold similarly for an arbitrary generator satisfying a logarithmic Sobolev inequality (1.5) in the abstract setting (and with $|\nabla U|^{2}$ replaced by $\left.\Gamma(U)\right)$.

The next proposition transfers the optimal (LS) inequality for $d x$ into a logarithmic Sobolev inequality for $\mu$. It is the key argument in the applications.

Proposition 3.1. For every smooth $f$ on $\mathbb{R}^{n}$ with $\int f^{2} d \mu=1$ and every $v>0$,

$$
\int f^{2} \log f^{2} d \mu \leq \frac{n}{2}\left(\log v-1+\frac{2}{n \pi \mathrm{e} v} \int|\nabla f|^{2} d \mu\right)+c(v)
$$

where

$$
c(v)=\sup _{x \in \mathbb{R}^{n}}\left(\frac{1}{4 \pi \mathrm{e} v}\left[2 \Delta U(x)-|\nabla U|^{2}(x)\right]+U(x)\right) .
$$

Proof. The proof is easy. We start with inequality (1.2)

$$
\int g^{2} \log g^{2} d x \leq \frac{n}{2} \log \left(\frac{2}{n \pi \mathrm{e}} \int|\nabla g|^{2} d x\right)
$$

where $\int g^{2} d x=1$. By concavity of the $\log$ function, for every $v>0$,

$$
\int g^{2} \log g^{2} d x \leq \frac{n}{2}\left(\log v-1+\frac{2}{n \pi \mathrm{e} v} \int|\nabla g|^{2} d x\right) .
$$

Set now $g^{2}=f^{2} \mathrm{e}^{-U}$. Then $\int f^{2} d \mu=1$ and

$$
\int|\nabla g|^{2} d x=\int|\nabla f|^{2} d \mu-\int f \nabla f \cdot \nabla U d \mu+\frac{1}{4} \int f^{2}|\nabla U|^{2} d \mu .
$$

It follows that

$$
\begin{aligned}
\int f^{2} \log f^{2} d \mu \leq \frac{n}{2}(\log v- & \left.+\frac{2}{n \pi \mathrm{e} v} \int|\nabla f|^{2} d \mu\right) \\
& +\int f^{2}\left(\frac{1}{4 \pi \mathrm{e} v}\left[2 \Delta U-|\nabla U|^{2}\right]+U\right) d \mu .
\end{aligned}
$$

Proposition 3.1 is established. 
Assume now we can find a concave increasing function $\Phi: \mathbb{R}^{+} \rightarrow \mathbb{R}^{+}$such that for every $u \geq 0$ there exists $v>0$ with

$$
\frac{n}{2}\left(\log v-1+\frac{2 u}{n \pi \mathrm{e} v}\right)+c(v) \leq \Phi(u)
$$

Then, by Proposition 3.1,

$$
\int f^{2} \log f^{2} d \mu \leq \Phi\left(\int|\nabla f|^{2} d \mu\right)
$$

for every smooth $f$ on $\mathbb{R}^{n}$ with $\int f^{2} d \mu=1$. We may then make use of the general theory recalled in Section 1 to obtain bounds on the heat kernel of L. Rather than to state a general result, let us inspect a number of examples drawn from [Kavian et al. (1993)] (see also [Rosen (1976)]).

Take, to start with, $U(x)=|x|^{\alpha}, \alpha>2$. Then, we may take

$$
c(v) \leq C v^{\alpha /(\alpha-2)}, \quad v \geq 1
$$

where $C>0$ only depends on $n$ and $\alpha$ and may vary from line to line below. The function

$$
\Phi(u)=C\left(1+u^{\alpha /(2 \alpha-2)}\right), \quad u \geq 0,
$$

will then satisfy (3.1). As a consequence, the bound (1.6) yields, for every $t>0$,

$$
\left\|P_{t}\right\|_{1, \infty} \leq \exp \left(\frac{C}{t^{\alpha /(\alpha-2)}}\right) .
$$

Similarly, if $U(x)=|x|^{2}\left(\log \left(1+|x|^{2}\right)\right)^{\alpha}, \alpha>1$, we obtain

$$
\left\|P_{t}\right\|_{1, \infty} \leq \exp \left[C t^{\alpha /(\alpha-1)} \exp \left(\frac{C}{t^{1 /(\alpha-1)}}\right)\right] .
$$

An interesting limiting case is obtained when $\alpha=1$ in the last example. It is known from [Kavian et al. (1993)] that the associated semigroup is not ultracontractive, but what is called immediately hypercontractive (i.e. $\left\|P_{t}\right\|_{1, \infty}=\infty$ but $\left\|P_{t}\right\|_{p, q}<\infty$ for every $\left.t>0,1<p<q<\infty\right)$. This is described by the bound

$$
\left\|P_{t}\right\|_{2, q} \leq \exp \left[\sqrt{1-\frac{1}{r}} \frac{C t^{2}}{\log ^{2} r} \exp \left(\frac{C \log r}{t}\right)\right]
$$

where $q>2, r=4 q^{2} /(q-1)$, which is obtained by the same method.

\section{ACKNOWLEDGEMENT}

We thank L. Saloff-Coste for helpful comments leading to Corollary 1.6. 


\section{REFERENCES}

Aubin, TH. (1982), Nonlinear analysis on manifolds. Monge-Ampère equations, Springer.

BAKRY, D. (1994), L'hypercontractivité et son utilisation en théorie des semigroupes, École d'Été de Probabilités de St-Flour, Lecture Notes in Math. 1581 1-114, Springer.

Bakry D., Coulhon T., Ledoux M., Saloff-Coste L. (1995), Sobolev inequalities in disguise, Indiana J. Math. 44 1034-1074.

CARLEN E. (1991), Superadditivity of Fisher's information and logarithmic Sobolev inequalities, J. Funct. Anal. 101 194-211.

Carlen E., Kusuoka S., Stroock D. (1987), Upperbounds for symmetric Markov transition functions, Ann. Inst. H. Poincaré 23 245-287.

Carlen E., Loss M. (1993), Sharp constant in Nash's inequality. Duke Math. J., International Math. Research Notices 7 213-215.

Carlen E., Loss M. (1995), Optimal smoothing and decay estimates for viscously damped conservation laws, with applications to the 2-D Navier-Stokes equation, Duke Math. J. 81 135-157.

Chavel I. (1993), Riemannian geometry : a modern introduction, Cambridge Univ. Press.

Davies E. B. (1989), Heat kernels and spectral theory, Cambridge Univ. Press.

Gross L. (1975), Logarithmic Sobolev inequalities, Amer. J. Math. 97 1061-1083.

Kavian O., Kerkyacharian G., Roynette (1993), Quelques remarques sur l'hypercontractivité, J. Funct. Anal. 111 155-196.

Li P. (1986), Large time behavior of the heat equation on complete manifolds with nonnegative Ricci curvature, Ann. Math. 124 1-21.

Li P., Yau S.T. (1986), On the parabolic kernel of the Schrödinger operator, Acta Math. $156153-201$.

LIEB E. (1990), Gaussian kernels have only Gaussian maximizers, Invent. math. 102 179-208.

Rosen J. (1976), Sobolev inequalities for weight spaces and supercontractivity, Trans. Amer. Math. Soc. 22 367-376.

Varopoulos N. (1985), Hardy-Littlewood theory for semigroups. J. Funct. Anal. 63 240-260.

D. BAkry, M. Ledoux: Département de Mathématiques, Laboratoire de Statistique et Probabilités assocí́ AU C.N.R.S., Université Paul Sabatier, 31062 TOULOUSE, FrancE. EMAIL: bakryecict.fr, ledouxecict.fr

D. Concordet: Unité de Biométrie, École VÉtérinaire de Toulouse, 31067 Toulouse, France et DÉpartement de Mathématiques, Laboratoire de Statistique ET Probabilités associé AU C.N.R.S., Université PAUL SABatier, 31062 Toulouse, France. Email: concordedcict.fr 